\title{
Hybrid Scheduling for Quality of Service Guarantee of Multimedia Data Flows in Software Defined Networks
}

\author{
Jun Huang, Liqian $\mathrm{Xu}$, \\ Mengxi Zeng, Huifang \\ Yan \\ SCIE, Chongqing Univ. of \\ Posts and Telecom. \\ Chongqing, China 400065 \\ jhuang@cqupt.edu.cn
}

\author{
Qiang Duan \\ IST Department \\ The Penn. State Univ. \\ Abington, PA 19001. \\ qduan@psu.edu
}

\author{
Cong-cong Xing \\ Math \& CS Department \\ Nicholls State Univ. \\ Thibodaux, LA 70310. \\ cong- \\ cong.xing@nicholls.edu
}

\begin{abstract}
Supporting diverse Quality of Service (QoS) performance for heterogeneous data flows generated by multimedia applications has been a challenging issue that is not fully addressed in the Internet. Software Defined Network, which decouples data forwarding and network control, offers a promising approach to QoS guarantee of multimedia flows in the future Internet. However, the currently available QoS mechanisms proposed for SDN have not fully considered the diversity in performance requirements of multimedia data flows. In order to tackle this challenge, we propose a hybrid scheduling scheme in this paper to combine priority queueing with Packet General Processor Sharing (PGPS) algorithm to provide QoS guarantee for multimedia applications in SDN. We apply network calculus to develop modeling and analysis techniques to evaluate the QoS performance of the proposed scheduling scheme. Both analytical and numerical results obtained in this paper show that the proposed scheme can provide QoS guarantee for meeting diverse requirements of multimedia applications.
\end{abstract}

\section{Categories and Subject Descriptors}

C.2.1 [Network Architecture and Design]: [Network Communications]

\section{General Terms}

Management, Design

\section{Keywords}

Packet scheduling, QoS, Software Defined Network, network calculus, performance analysis

\section{INTRODUCTION}

Software Defined Network (SDN) is an emerging networking paradigm that is expected to have a significant impact on fundamental architecture of the next generation networks. SDN is a programmable network approach that supports separation of control and forwarding planes via standardized interfaces [1]. Decoupling forward and control planes in SDN enables a logically centralized controller to obtain a global view of network states for optimal network control. The centralized controller also makes it possible for upper layer applications to program and configure network infrastructure to better satisfy their requirements. Therefore, SDN offers a promising approach to significantly improving network performance for supporting diverse applications; thus playing a crucial role in the future Internet.

Multimedia applications, for example WebTV, video on demand, online gaming, and video conference, are becoming exceedingly popular over the Internet. Multimedia applications bring in more challenges to network control due to their requirements for more bandwidth, little or no packet drop, and low packet delay and delay variation. In addition, the diversity in multimedia applications, which have different traffic characteristics and performance requirements, further complicates the network control for supporting both multimedia applications and best-effort traffic coexisting in the Internet while achieve full utilization of network resources as well.

QoS provisioning for supporting the diverse requirements of multimedia applications in the Internet has been a challenging open issue in the Internet. The centralized control plane and flow-based packet forwarding enabled by SDN offer a promising approach to providing the required QoS guarantee to support multimedia applications in the future Internet. Research progress has been made toward QoS mechanism in SDN for supporting multimedia applications.

Currently OpenFlow (OF) is the most popular protocol between the controller and switches in SDN [2]. Solutions to QoS guarantee through adjusting flow routing in OF have been proposed. An optimization model is designed in [3] for distinguish QoS-required traffic from best-effort traffic for flow routing. The model is extended in [4] to allow service provider to configure routing calculation through an API. Although flow routing definitely plays a significant role in QoS guarantee, packet forwarding control is also needed in OF switches to actually enforce the QoS policy for each traffic flow. QoS architecture presented in [5] adds rate-limiter 
and queue mapping mechanism to map traffic flows to specific rate-limiters and priority queues in OF switches. The QoSFlow framework proposed in [6] controls multiple packet schedulers in each OF switch for improving network QoS capability. The work of [5] and [6] integrate configuration of queue management into OpenFlow protocol. In order to enhance configuration of OF switches, OF-CONFIG [7] has been developed by ONF as an auxiliary protocol of the OpenFlow protocol. In [8], the authors presented an autonomic management mechanism that can configure various QoS features of queuing and scheduling functions in $\mathrm{OF}$ switches through OF-CONFIG protocol.

Although encouraging progress toward QoS provisioning in SDN has been made, the aforementioned works have not fully addressed the challenges brought in by the heterogeneous traffic flows of multimedia applications with diverse performance requirements. The QoS control in SDN, more specifically queuing and scheduling mechanisms in OF switches should be able to not only meet the performance requirements of heterogeneous multimedia data flows but also achieve high utilization of network resources by fairly sharing spare bandwidth among best-effort flows. A single level scheduling scheme, such as Packet General Processor Share (PGPS) or Weighted Fair Queuing (WFQ), cannot fully meet such requirements.

In order to address this challenging issue, we propose a hybrid scheduling scheme that combines priority queues and PGPS scheduling for QoS guarantee of multimedia data flows in SDN. In order to obtain a deep insight about QoS capability of the hybrid scheduling scheme, we apply network calculus in this paper to develop a model and analysis technique to evaluate delay and backlog performance of this scheme. Network calculus is a new mathematical tool in the field of computer network modeling and performance analysis that offers effective computation for obtaining upper bounds of performance parameters such as queueing delay and backlog. Application of network calculus in this paper makes the developed modeling and analysis techniques general and flexible to be applicable to QoS control in OF switches with diverse implementations to support various heterogeneous data flows of multimedia applications. Specifically we present the following contributions in this paper:

- We propose a hybrid scheduling model for the QoS guarantee for heterogeneous data flows.

- We analyze performance of the proposed hybrid scheduling model using network calculus technique for the single-hop case.

- We determine performance boundaries guaranteed by the proposed scheduling scheme to heterogeneous data flows, including the worst-case packet delay and queue backlog.

- We conduct extensive numerical analysis to evaluate the effectiveness of the proposed scheme and investigate the impacts of different queueing and scheduling parameter settings on performance of the model.

The rest of this paper is organized as follows. Preliminaries of network calculus theory are provided in Sections 2. The hybrid scheduling model is proposed in Section 3 and its performance analysis is given in Section 4. Numerical analysis results are provided in Section 5. We draw conclusions and discuss possible future work in Section 6 .

\section{PRELIMINARIES}

In this section, we briefly present the main definitions and notations in Network Calculus that will be used in the rest of the paper. The theory of network calculus was created by Chang [17] and Cruz [18] and then extended by others (e.g. $[19,20,6])$. The following definitions will be needed in dealing with the multi-priority of traffic flows.

Definition 1. (Wide-Sense Increasing Sequences). If $F=$ $\{f(t) \mid f(t)=0, \forall t<0 ; f(0) \geq 0 ; f(s) \leq f(t), \forall s \leq t, s, t \in$ $[0,+\infty]\}$, we say that $F$ is wide-sense increasing sequences.

Definition 2. (Min-Plus Convolution). Let $f$ and $g$ be two functions or sequences of $F$. The min-plus convolution of $f$ and $g$ is the function $(f \otimes g)(t)=\inf _{0 \leq u \leq t}[f(u)+g(t-u)]$, if $t<0, f \otimes g=0$.

Definition 3. (Arrival Curve). Given a wide-sense increasing function $\alpha$ defined for $t \geq 0$, we say that a flow $R$ is constrained by $\alpha$ if and only if for all $s \leq t$ such that $R(t)-R(s) \leq \alpha(t-s)$. We say that $R$ has $\alpha$ as an arrival curve, or also that $R$ is $\alpha$-smooth.

Since the arrival curve is often regulated by the leaky buckets function, we will use affine arrival curves $\gamma_{r, b}$, defined by: $\gamma_{r, b}(t)=r t+b$ for $t>0$ and 0 otherwise. Parameters $b$ and $r$ are called the burst tolerance and rate. Note that the leaky buckets function herein is used for illustration, other arrival curves can be employed for performance analysis below in a similar way.

Definition 4. (Service Curve). Consider a system $S$ and a flow through $S$ with input and output function $R$ and $R^{*}$. We say that $S$ offers to the flow a service curve $\beta$ if and only if $\beta$ is wide sense increasing, $\beta(0)=0$ and $R^{*} \geq R \otimes \beta$.

The service curve that a system offers to a flow gives the lower bound of the service capacity that the system guarantees to the flow. Since a service curve is given as a function of time that is defined by the relationship between arrival and departure traffic of a flow at the system, it is agnostic to system implementations; thus providing a general approach to characterizing the service capability of any system.

Assume a flow constrained by the arrival curve $\alpha$, traverses a system that offers a service curve $\beta$, then

Definition 5. (Delay Bound). The delay bound $P(t)$ is expressed as

$$
\begin{gathered}
P(t) \leq h(\alpha, \beta), \\
h(\alpha, \beta)=\sup _{t \geq 0}\{\inf \{d \geq 0: \alpha(t) \leq \beta(t+d)\}\} .
\end{gathered}
$$




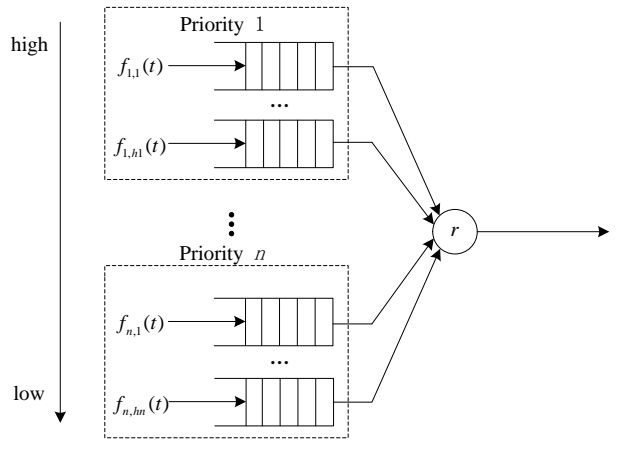

Figure 1: Hybrid Scheduling Model.

DeFinition 6. (Backlog Bound). The backlog bound $Q(t)$ is expressed as

$$
Q(t)=R(t)-R^{*}(t) \leq \sup _{s \geq 0}\{\alpha(s)-\beta(s)\} .
$$

\section{HYBRID SCHEDULING MODEL}

We propose a hybrid scheduling model for multimedia flows in SDN, which combines the PGPS algorithm and the preemptive priority queueing algorithm. We also use the leaky bucket to shape the traffic, ensuring that the low-priority data also has an upper bounded determined performance, and avoiding the starvation phenomenon.

The scheme of hybrid scheduling is depicted in Figure 1, and the frequently used notations associated with the network calculus are shown in Table 1 . There are $n$ different types of priorities and for each priority level there are $h_{i}$ flows with the same level of priority. This configuration is used to differentiate the heterogeneous flows in SDN and to improve the QoS for multimedia flow communications.

When data flows arrive at a node, they will be classified by their priorities first. Preemptive scheduling is applied to high-priority flows to meet their real-time transmission requirements, and PGPS is applied to the flows within the same rank of priority to ensure the fairness. Thus, both service distinctions and service fairness are handled in the hybrid scheduling model.

\section{END-TO-END PERFORMANCE ANALY- SIS}

We now turn our attention to the performance analysis of the proposed hybrid scheduling model. We use the network calculus theory to quantitatively analyze the end-to-end communication performances of data of different types in the mode, with a focus on the worst-case scenario of delay upper bound and backlog upper bound. As shown in Figure 1, there are $n$ prioritized queues in the system with 1 being the highest priority and $n$ the lowest. When a flow arrives at the queue and cannot be immediately serviced, we assume that this flow will be saved in sufficiently-larger buffers so that no packets will be dropped. Also, we assume that all services will be conducted at rate $r$. Our discussion of the end-to-end deterministic performance analysis is split into two cases: single-hop and multi-hop.

\subsection{Service Curve}

Table 1: Frequently Used Notations.

\begin{tabular}{|c|c|}
\hline$f_{i, m}$ & The $m$-th data flow with priority $i$ \\
\hline$\alpha_{i, m}$ & $\begin{array}{l}\text { Arrival curve of the } m \text {-th data flow with } \\
\text { priority } i\end{array}$ \\
\hline$r_{i, m}$ & Rate of the $m$-th data flow with priority $i$ \\
\hline$b_{i, m}$ & Burst of the $m$-th data flow with priority $i$ \\
\hline$\beta(t)$ & The service curve \\
\hline$\beta_{R_{i, m}, T_{i, m}}(t)$ & $\begin{array}{l}\text { Service curve of the } m \text {-th data flow with } \\
\text { priority } i\end{array}$ \\
\hline$w_{i, m}$ & $\begin{array}{l}\text { Weight of the } m \text {-th data flow with priority } \\
i\end{array}$ \\
\hline$L_{i, \max }$ & Maximum packet length of priority $i$ \\
\hline$L_{i, \operatorname{m}, \max }$ & $\begin{array}{l}\text { Maximum packet length of the } m \text {-th data } \\
\text { flow with priority } i\end{array}$ \\
\hline$R_{i}(t), R_{i}^{*}(t)$ & $\begin{array}{l}\text { Input and output cumulative functions of } \\
\text { a flow with priority } i\end{array}$ \\
\hline$s_{i}$ & Backlog start time of a flow with priority $i$ \\
\hline$D_{i, m}(t)$ & $\begin{array}{l}\text { Delay bound of the } m \text {-th data flow with } \\
\text { priority } i\end{array}$ \\
\hline$Q_{i, m}(t)$ & $\begin{array}{l}\text { Backlog bound of the } m \text {-th data flow with } \\
\text { priority } i\end{array}$ \\
\hline
\end{tabular}

The following theorem presents the service curve.

ThEOREM 1. Suppose that data flow $f_{i, m}$ is the $m$-th data flow with priority $i$, and $f_{i, m}$ is constrained by the arrival curve $\alpha_{i, m}(t)=r_{i, m} t+b_{i, m}, 1 \leq i \leq n$ and $1 \leq m \leq h_{i}$. Also, assume that the total service curve is $\beta(t)=r[t-0]^{+}$ . Then, the service curve $\beta_{R_{i, m}, T_{i, m}}(t)$ for $f_{i, m}$ is

$$
\beta_{R_{i, m}, T_{i, m}}(t)=R_{i, m}\left[t-T_{i, m}\right]^{+},
$$

where $R_{i, m}=w_{i, m} \cdot \frac{R_{i, m}^{\prime}}{\sum_{k=1}^{h_{i}} w_{i, k}}, T_{i, m}=T_{i, m}^{\prime}+\frac{L_{i, \max }}{R_{i, m}^{\prime}}+\frac{L_{i, m, \max }}{R_{i, m}}$, $R_{i, m}^{\prime}=r-\sum_{i<j} \sum_{l=1}^{h_{j}} r_{j, l}$, and $T_{i, m}^{\prime}=\frac{\sum_{i<j} \sum_{l=1}^{h_{j}} b_{j, l}}{R_{i, m}^{\prime}}$.

Proof. Let $s_{i}$ be the time that priority $i$ data flow start to backlog, $\left(s_{i}, t\right]$ be the busy period. Thus the node output over this period of time is $r\left(t-s_{i}\right)$. With the preemptive scheduling, data in the highest priority queue will be serviced first, and when a higher priority data flow arrives, the service for low priority data will be interrupted immediately and the service will be transferred to the higher priority data. As a result, starting from $s_{i}$, the data with priority $i$ will have to wait, at most, for all higher-priority services to finish. Therefore we have

$$
R_{i}^{*}(t)-R_{i}^{*}\left(s_{i}\right)=r\left(t-s_{i}\right)-\sum_{i<j}\left(R_{j}(t)-R_{j}\left(s_{i}\right)\right),
$$

where $R_{i}^{*}(t)$ and $R_{j}(t)$ denote the output accumulation function of the flow with priority $i$ and input accumulation function of the flow with priority $j$ over the time interval $[0, t]$, respectively. We also have

$$
\begin{aligned}
0 & \leq R_{i}^{*}(t)-R_{i}^{*}\left(s_{i}\right)=R_{i}^{*}(t)-R_{i}\left(s_{i}\right) \\
& \leq R_{i}(t)-R_{i}\left(s_{i}\right) \leq \alpha_{i}\left(t-s_{i}\right) .
\end{aligned}
$$




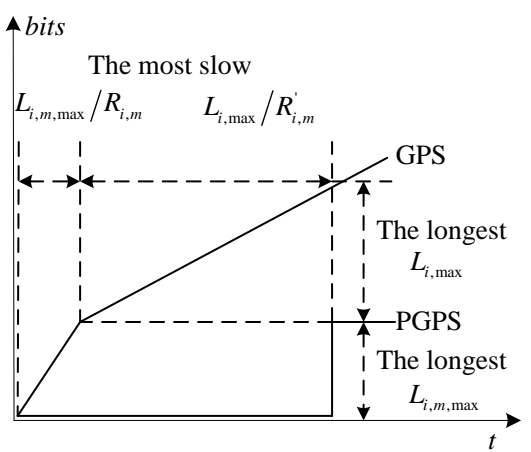

Figure 2: Cumulative function of PGPS and GPS.

Combining equation (5) and inequality (6) give us the following

$$
\begin{aligned}
R_{i}^{*}(t) & \geq R_{i}\left(s_{i}\right)+K_{i}\left(t-s_{i}\right) \\
& \geq \inf _{0 \leq s_{i} \leq t}\left\{R_{i}\left(s_{i}\right)+K_{i}\left(t-s_{i}\right)\right\} \\
& =\left(R_{i} \otimes K_{i}\right)(t),
\end{aligned}
$$

where $K_{i}\left(t-s_{i}\right)=\left[r\left(t-s_{i}\right)-\sum_{i<j} \sum_{l=1}^{h_{j}} \alpha_{j, l}\left(t-s_{i}\right)\right]^{+}$.

The service curve of data flow with priority $i$ would be $K_{i}(t-$ $\left.s_{i}\right)$, if $K_{i}\left(t-s_{i}\right)$ is a wide-sense increasing function. Hence, we can represent the service curve as a rate-latency function

$$
\beta_{i}(t)=R_{i, m}^{\prime}\left[t-T_{i, m}^{\prime}\right]^{+},
$$

where $R_{i, m}^{\prime}=r-\sum_{i<j} \sum_{l=1}^{h_{j}} r_{j, l}$ and $T_{i, m}^{\prime}=\frac{\sum_{i<j} \sum_{l=1}^{h_{j}} b_{j, l}}{R_{i, m}^{\prime}}$.

As a fair service scheduling algorithm for heterogeneous flows, PGPS primarily simulates the ideal GPS scheduling algorithm and operates in a similar manner with GPS. In other words, when the data flows arrive, PGPS will schedule them in the same way as GPS does. By comparing the sending time of PGPS and of GPS, we can find that the sending rate of PGPS may be slower than that of GPS, with one data packet being delayed at most whose maximum size is $L_{i, \max }[6]$. Since we have obtained that the minimum service rate for priority flows is $R_{i, m}^{\prime}$, the finishing time for PGPS is at most the finishing time of GPS plus $\frac{L_{i, \max }}{R_{i, m}^{\prime}}$. It can be seen in Figure 2 that when the sending time of PGPS is later than the sending time of GPS, the cumulative function of PGPS would be at most $L_{i, m, \text { max }}$ packets less than that of GPS. Considering that the slowest service rate for data flow $L_{i, m, \max }$, is $R_{i, m}=w_{i, m} \cdot \frac{R_{i, m}^{\prime}}{\sum_{k=1}^{h_{i}} w_{i, k}}$ [13], which is the same as that for GPS, we can see that the delay function under the worst circumstance is $\frac{L_{i, \max }}{R_{i, m}^{\prime}}+\frac{L_{i, m, \max }}{R_{i, m}}$.

In summary, the minimum service rate of data flow $f_{i, m}$ is $R_{i, m}$ and the latency is $T_{i, m}=T_{i, m}^{\prime}+\frac{L_{i, \max }}{R_{i, m}^{\prime}}+\frac{L_{i, m, \max }}{R_{i, m}}$. Hence the service curve of data flow $f_{i, m}$ is $\beta_{R_{i, m}, T_{i, m}}(t)=$ $R_{i, m}\left[t-T_{i, m}\right]^{+}$. This completes the proof.

\subsection{Performance Bounds}

Using the service curves obtained in the previous section, we can derive the delay upper bound and the backlog upper bound. To this end, we assume that the arrival rate and the service rate satisfy the constraint $\sum_{i=1}^{n} \sum_{m=1}^{h_{i}} r_{i, m} \leq r$ which is depicted in Figure 3, where Figure 3(a) shows the case where the constraint is satisfied, and Figure 3(b) shows the case where constraint is not satisfied. Clearly, it can be seen from Figure 3 that performance upper bound exists if and only if the constraint is satisfied (otherwise the upper bound will be infinity).

THEOREM 2. The delay upper bound of data flow $f_{i, m}$, in the case of single-hop, is given as follows:

$$
D_{i, m}(t)=\frac{b_{i, m}+L_{i, m, \max }}{\frac{w_{i, m}}{\sum_{k=1}^{h_{i}} w_{i, k}}\left(r-\sum_{i<j} \sum_{l=1}^{h_{j}} r_{j, l}\right)}+\frac{\sum_{i<j} \sum_{l=1}^{h_{j}} b_{j, l}+L_{i, \max }}{r-\sum_{i<j} \sum_{l=1}^{h_{j}} r_{j, l}} .
$$

Proof. By Theorem 1, we know that the delay upper bound is the horizontal deviation between the arrival curve $\alpha_{i, m}(t)=r_{i, m} t+b_{i, m}$ and the service curve $\beta_{R_{i, m}, T_{i, m}}(t)$. Thus,

$$
\begin{aligned}
& D_{i, m}(t)=h\left(\alpha_{i, m}, \beta_{R_{i, m}, T_{i, m}}\right) \\
& =\sup _{t \geq 0}\left\{\operatorname { i n f } \left\{d \geq 0: \alpha_{i, m}(t)\right.\right. \\
& \left.\left.\leq \beta_{R_{i, m}, T_{i, m}}(t+d)\right\}\right\} \\
& =\inf \left\{d \geq 0: \alpha_{i, m}(0) \leq \beta_{R_{i, m}, T_{i, m}}(d)\right\} \\
& =\inf \left\{d \geq 0: b_{i, m} \leq R_{i, m} \cdot d-\frac{R_{i, m} \cdot \sum_{i<j} \sum_{l=1}^{h_{j}} b_{j, l}}{R_{i, m}^{\prime}}\right. \\
& \left.-\frac{R_{i, m} \cdot L_{i, \max }}{R_{i, m}^{\prime}}-L_{i, m, \max }\right\} \\
& =\inf \left\{d \geq 0: d \geq \frac{b_{i, m}+L_{i, m, \max }}{R_{i, m}}\right. \\
& \left.+\frac{\sum_{i<j} \sum_{l=1}^{h_{j}} b_{j, l}+L_{i, \max }}{R_{i, m}^{\prime}}\right\} \\
& =\frac{b_{i, m}+L_{i, m, \max }}{\frac{w_{i, m}}{\sum_{k=1}^{h_{i}} w_{i, k}}\left(r-\sum_{i<j} \sum_{l=1}^{h_{j}} r_{j, l}\right)}+\frac{\sum_{i<j} \sum_{l=1}^{h_{j}} b_{j, l}+L_{i, \max }}{r-\sum_{i<j} \sum_{l=1}^{h_{j}} r_{j, l}} .
\end{aligned}
$$

This completes the proof.

THEOREM 3. The backlog upper bound of data flow $f_{i, m}$ 


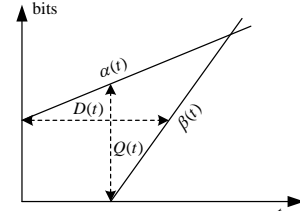

(a)

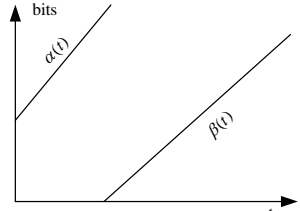

(b)
Figure 3: Constraint of arrival and service curves: (a) constraint satisfied, (b) constraint unsatisfied.

, in the case of single-hop, is given as follows:

$$
\begin{aligned}
Q_{i, m}(t)=b_{i, m}+r_{i, m} \cdot\left[\frac{\sum_{i<j} \sum_{l=1}^{h_{j}} b_{j, l}+L_{i, \max }}{r-\sum_{i<j} \sum_{l=1}^{h_{j}} r_{j, l}}\right. \\
\left.+\frac{L_{i, m, \max }}{\frac{w_{i, m}}{\sum_{k=1}^{h_{i}} w_{i, k}}\left(r-\sum_{i<j} \sum_{l=1}^{h_{j}} r_{j, l}\right)}\right] .
\end{aligned}
$$

Proof. By Theorem 2, we know that the backlog upper bound is the vertical deviation between the arrival curve $\alpha_{i, m}(t)=r_{i, m} t+b_{i, m}$ and the service curve $\beta_{R_{i, m}, T_{i, m}}(t)$. Thus,

$$
\begin{aligned}
& Q_{i, m}(t)=R_{i, m}(t)-R_{i, m}^{*}(t) \\
& \leq \sup _{t \geq 0}\left\{\alpha_{i, m}(t)-\beta_{R_{i, m}, T_{i, m}}(t)\right\} \\
&=\alpha_{i, m}\left(T_{i, m}\right)-\beta_{R_{i, m}, T_{i, m}}\left(T_{i, m}\right) \\
&=\alpha_{i, m}\left(T_{i, m}\right) \\
&=b_{i, m}+r_{i, m} \cdot\left[\frac{\sum_{i<j} \sum_{l=1}^{h_{j}} b_{j, l}+L_{i, \max }}{r-\sum_{i<j} \sum_{l=1}^{h_{j}} r_{j, l}}\right. \\
&+\frac{L_{i, m, \max }}{w_{i, m}}\left(r-\sum_{i<j} \sum_{l=1}^{h_{j}} r_{j, l}\right) \\
& \sum_{k=1}^{h_{i}} w_{i, k}
\end{aligned}
$$

This completes the proof.

\section{NUMERICAL RESULTS}

In the previous section, we obtained the service curve for the single-hop case, and proved that the end-to-end delay upper bound and backlog upper bound, respectively. In this section, we evaluate those performance bounds and discuss various aspects that may impact these performance bounds through numerical experiments. This allows us to have an in-depth understanding for the data delivery performance of our model. In these numerical experiments, we assume that there are three data flows, $f_{1,1}, f_{1,2}$, and $f_{2,1} . f_{2,1}$ has higher priority than that of $f_{1,1}$ and of $f_{1,2}$. Also, according to [21], we assume that the maximum size of packet of a data flow is 1500 byte, and the burst data transfer parameter is $1.1 \mathrm{Mb}$. In PGPS, $w_{i, k}$ of data flows are $0.3,0.2$, and 0.5. Note that preemptive scheme is used in the proposed hybrid scheduling model, and that lower-priority flows can be served only if there are no higher-priority flows being present, thus $w_{1,1}$ and $w_{1,2}$ are proportionally increased to 0.6 and 0.4 in our model.

Figures 5(a) and 5(b) show the impact of the arrival rate and the service rate of $f_{2,1}$ on the end-to-end delay upper bounds in single-hop case. Figure 5(c) shows the delay upper bounds in hybrid scheduling and in PGPS under different service rates. We can see, in Figure 5, that the end-to-end delay upper bound of high-priority data flows is lower than that of low-priority data flows. This means that flows with high-priority have better delay guarantee than flows with low-priority. Specifically, Figure 5(a) suggests that as the arrival rate of high-priority flows increases, the delay upper bounds of low-priority flows also increase, and with a growing rate. Figure 5(b) suggests that when the arrival rates of flows are fixed, the delay upper bounds of all flows decrease as the service rate increases, with the low-priority flows being affected the most; however, the decreasing rate gets consistently smaller as the service rate gets larger. Figure 5(c) depicts the comparison of delay upper bounds between PGPS and our model. As shown in this figure, the delay upper bounds of all flows in our model are less than that of PGPS, with $f_{2,1}$ being especially notable when the service rate is relatively small. This situation indicates that our hybrid scheduling model can provide better service guarantee to real-time data transmission.

Figures 6(a) and 6(b) respectively illustrate how the backlog upper bounds of flows are affected by arrival rate of $f_{2,1}$ and the service rate of node in single-hop case. Figure 6(c) compares the backlog upper bounds of flows in our model with that in PGPS, with respect to changes in the service rate. Obviously, we can see in these figures that the backlog upper bounds of high-priority flows are lower than that of low-priority flows. More specifically, Figure 6(a) indicates that the arrival rate of $f_{2,1}$ has significant impact on the backlog upper bounds of data flows with lower priorities in that the backlog upper bounds increase as the arrival rate of $f_{2,1}$ increases. Figure 6(b) shows a similar situation to that the delay upper bounds discussed earlier in the sense that the backlog upper bound of all flows decrease as the service rate increases. Also, this impact becomes more obvious when the sum of all arrival rates is close to the service rate. Figure 6(c) clearly shows that the backlog upper bounds of all flows in our model are lower than that of PGPS, suggesting that our model is superior to PGPS. Interestingly, note that in PGPS the backlog upper bound of $f_{2,1}$ is higher than that of the other two flows, and this is because $f_{2,1}$ has a higher arrival rate than the other two flows. This fact evidently shows that the arrival rate of a flow will have an impact on its backlog upper bound.

Summarizing discussions in this section, we see that arrival rate and service rate both have a substantial impact on flowsąr deterministic performance upper bound. The delay upper bound and backlog upper bound of low priority flows depend not only on the service rate but also on the arrival rate of higher priority flows. Under the same setting, flows with high priority perform clearly better than flows with low priority. Moreover, the delay upper bounds and backlog upper bounds of data flows exhibited in our hybrid scheduling 


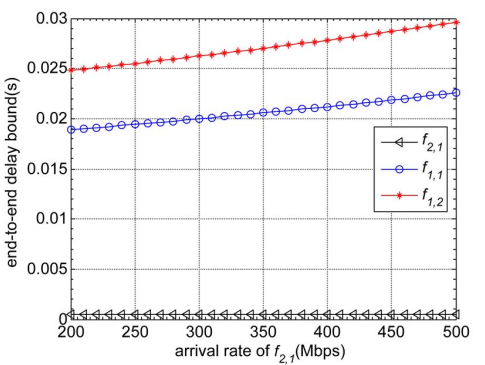

(a) $r=2$ Gbps, $r_{1,1}=r_{1,2}=$ $200 M$ bps,$r_{2,1} \in[200,500]$ Mbps

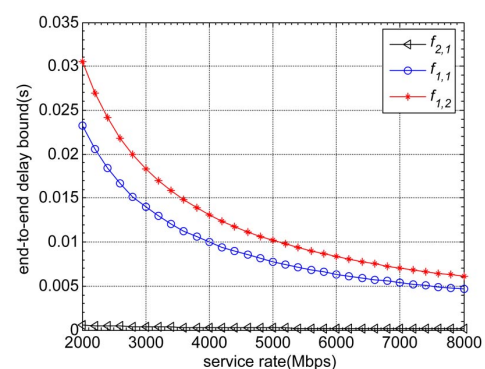

(b) $r_{2,1}=500$ Mbps, $r_{1,1}=r_{1,2}=$ 200Mbps, $r \in[2000,8000]$ Mbps

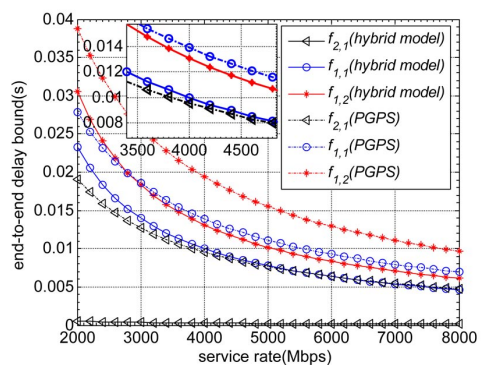

(c) $r_{2,1}=500 \mathrm{Mbps}, r_{1,1}=r_{1,2}=$ $200 M$ bps, $r \in[2000,8000]$ Mbps

Figure 4: Delay upper bounds in the single-hop case.

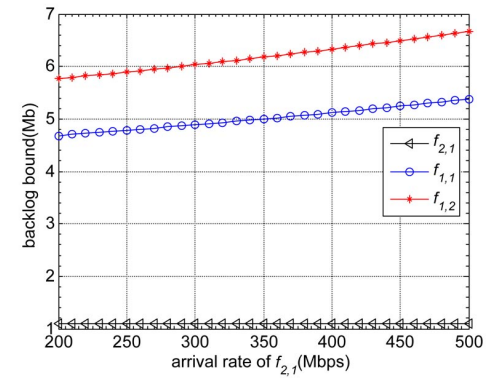

(a) $r=2$ Gbps, $r_{1,1}=r_{1,2}=$ $200 \mathrm{Mbps}, r_{2,1} \in[200,500] \mathrm{Mbps}$

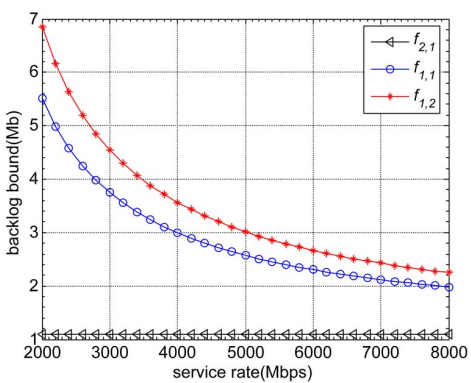

(b) $r_{2,1}=500 \mathrm{Mbps}, r_{1,1}=r_{1,2}=$ 200 Mbps, $r \in[2000,8000]$ Mbps

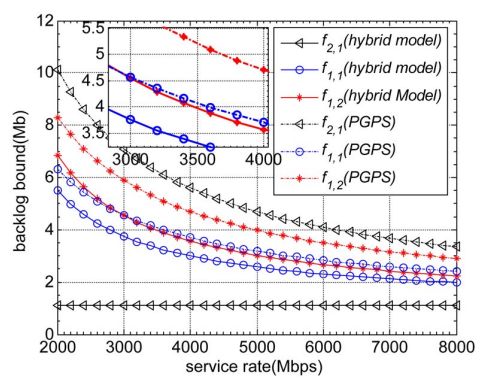

(c) $r_{2,1}=500 \mathrm{Mbps}, r_{1,1}=r_{1,2}=$ $200 \mathrm{Mbps}, r \in[2000,8000] \mathrm{Mbps}$

Figure 5: Backlog upper bounds in the single-hop case.

model are lower than that in PGPS, in both single-hop and multi-hop cases.

\section{CONCLUSIONS}

To deal with the issue of real-time transmissions of data flows and the fairness of scheduling of data flows of the same priority in SDN, we proposed a hybrid scheduling model which combines the essence of PGPS and preemptive scheduling. Using the theory of Network Calculus, we established an end-to-end deterministic performance analysis for the proposed hybrid scheduling model in single-hop and multi-hop cases, which include service curve, delay, and backlog. Our analysis indicates that the hybrid model can provide deterministic guaranteed QoS for heterogeneous flows. We further evaluated the model through numerical experiment case studies. The evaluation result demonstrated that our hybrid scheduling model is superior to PGPS in terms of both delay upper bound and backlog upper bound. We plan to, as future work, further the study of the multi-hop case and the hybrid scheduling model to deliver improved QoS guarantees for various types of data transmissions.

\section{ACKNOWLEDGMENTS}

This research is supported by NSFC under grants 61309031 and 61272400, Postdoctoral Science Foundation of China (Grant No. 2014M551740), Program for Innovation Team Building at Institutions of Higher Education in Chongqing under grant KJTD201310, NSF of Chongqing under grant cstc2013jcyjA40026, S\&T Research Program of Chongqing Municipal Education Commission under grant KJ130523, and CQUPT Research Fund for Young Scholars under grant
A2012-79.

\section{REFERENCES}

[1] L. Atzori, A. Iera, and G. Morabito, The internet of things: A survey,Computer Networks, vol. 54 , no. 15 , pp. $2787-2805,2010$

[2] R. Duan, X. Chen, and T. Xing, A QoS architecture for Iot, in Internet of Things (iThings/CPSCom), in Proceedings of the 4th International Conference on Cyber, Physical and Social Computing, 2011, pp. 717-720.

[3] M. Chui, M. Loffler, and R. Roberts, The internet of things, McKinsey Quarterly, vol. 2, pp. 1-9, 2010.

[4] L. Zhang, J. Yu, and X. Deng, Modelling the guaranteed qos for wireless sensor networks: a network calculus approach, EURASIP Journal on Wireless Communications and Networking, vol. 2011, no. 1, pp. 1-14, 2011.

[5] S. Zolhavarieh and M. Barati, Quality of service in wireless sensor networks (qos in wsn), in 2012 International Conference on Graphic and Image Processing, 2013, pp. 87684R-87684R.

[6] J.-Y. Le Boudec and P. Thiran, Network calculus: a theory of deterministic queuing systems for the internet. Springer, 2001, vol. 2050.

[7] P. Jurcik, R. Severino, A. Koubaa, M. Alves, and E. Tovar, Real-time communications over cluster-tree sensor networks with mobile sink behaviour, in Proceedings of the 14th IEEE International Conference on Embedded and Real-Time Computing Systems and Applications (RTCSA 08), 2008, pp. 
401-412.

[8] A. Koubaa, M. Alves, and E. Tovar, Modeling and worst-case dimensioning of cluster-tree wireless sensor networks, in Proceedings of the27th IEEE International Real-Time Systems Symposium (RTSS 06), 2006, pp. 412-421.

[9] L. Bisti, L. Lenzini, E. Mingozzi, and G. Stea, Numerical analysis of worst-case end-to-end delay bounds in fifo tandem networks, Real-Time Systems, vol. 48, no. 5, pp. 527-569, 2012.

[10] L. Bisti, L. Lenzini, E. Mingozzi, et al, Deborah: a tool for worst-case analysis of fifo tandems, in Proceedings of the 4th International Symposium on Leveraging Applications, LNCS 6415, 2010, pp. 152-168.

[11] J. B. Schmitt, F. A. Zdarsky, and L. Thiele, A comprehensive worst-case calculus for wireless sensor networks with in-network processing,in Proceedings of the 28th IEEE International Real-Time Systems Symposium (RTSS 2007), 2007, pp. 193-202.

[12] Y. Hua and X. Liu, Scheduling design and analysis for end-to-end heterogeneous flows in an avionics network, in Proceedings of IEEE INFOCOM 2011, pp. $2417=2425$.

[13] J. Schmitt, P. Hurley, M. Hollick, and R. Steinmetz, Per-flow guarantees under class-based priority queueing, in Proceedings of 2003 IEEE Global Telecommunications Conference (GLOBECOM 03), vol. 7,2003 , pp. $4169=4174$.

[14] A. Bouillard and A. Junier, Worst-case delay bounds with fixed priorities using network calculus, in Proceedings of the 5th International ICST Conference on Performance Evaluation Methodologies and Tools, 2011, pp. 381-390.

[15] R. Duan, X. Chen, and T. Xing, A Qos architecture for Iot, in Internet of Things (iThings/CPSCom), in Proceedings of the 4th International Conference on Cyber, Physical and Social Computing, 2011, pp. 717-720.

[16] J. Huang, Z. Xiong, Y. Liu, Q. Duan, Y. He, J. Lv, and J. Wang, Multi-priority scheduling using network calculus: Model and analysis, in Proceedings IEEE GLOBECOM 2013, 2013.

[17] C.S. Chang, Stability, queue length, and delay of deterministic and stochastic queueing networks, Automatic Control, IEEE Transactions on, vol. 39, no. 5, pp. 913-931, 1994.

[18] R. L. Cruz, A calculus for network delay. IEEE Transactions on Information Theory, vol. 37, no. 1, pp. 114-144, 1991.

[19] R. Agrawal, R. L. Cruz, C. Okino, and R. Rajan, Performance bonds for flow control protocols, IEEE/ACM Transactions on Networking (TON), vol. 7, no. 3, pp. 310-323, 1999.

[20] Y. Jiang and Y. Liu, Stochastic network calculus. Springer, 2008.

[21] H. Li, X. Liu, W. He, J. Li, and W. Dou, End-to-end delay analysis in wireless network coding: A network calculus-based approach, in 2011 31st International Conference on Distributed Computing Systems (ICDCS), 2011, pp. 47-56. 